Editorial

\title{
Smart computing for large scale visual data sensing and processing
}

Smart computing is an emerging multidisciplinary area, aiming to use computing technology to design smart methods, build smart systems, and make human life better. Visual signal plays the most important role in the communication and interaction between human and the surrounding world, while the past decade has witnessed the rapid development of digital imaging and transmission technologies. It is estimated that by 2015 the U.S. consumers will capture more than 100 billion digital pictures annually. In many practical vision applications, how to smartly collect the desired data and how to design smart algorithms to analyze the collected large amount of visual data have become very challenging issues. Meanwhile, the advancement of cloud computing, social computing, machine learning and artificial intelligence technologies are bringing smart computing to a newer dimension and improving our ways of living.

This special issue aims to present recent advances in smart computing for large scale visual data sensing and processing. Specifically, it focuses on novel methods for the smart acquisition, segmentation, feature representation, recognition and retrieval with their use to security, robotics, remote sensing, etc. It provides an effective forum for researchers and engineers from a broad range of disciplines to exchange their creative ideas, share recent research advances, and identify future directions in response to grand challenges in intelligent computing technologies for large scale vision problems.

This special issue received 23 submissions, confirming that smart computing is an active topic for large scale visual data sensing and processing. Each paper was examined by the guest editors to ensure that it falls into the scope of the special issue, and was reviewed by at least two reviewers. After more than two rounds of reviews, finally 10 papers were accepted for publication. The accepted papers cover several major methods (i.e., sparse and low rank models, dictionary learning) and applications fields (i.e., low level vision, emotion and behavior analysis, and image retrieval) of smart computing.

Sparse and low rank models have achieved great successes and remained an active topic in many vision applications. The paper "Low-rank+Dual" Model Based Dimensionality Reduction by Wang et al. studies the problem of matrix decomposition using low rank model. To minimize the correlation between the low rank matrix and the error matrix, the authors adopted a "low-rank+dual" model and further simplified it into the $" l_{1}+l_{\infty}$ " minimization problem. Wang et al. further used the surveillance video and face dataset to validate the superiority of their method. The paper Structured Occlusion Coding for Robust Face Recognition by Wen et al. aims to improve the robustness of sparse representation based classification (SRC) against occlusion. Considering the predictability of real face occlusion, the authors suggested a structured occlusion coding (SOC) method by seeking structured sparse solution on occlusion-appended dictionary, and obtained better robustness to real occlusion on the CAS-PEAL and AR face datasets.

Dictionary learning seeks a dictionary to enhance the representative or discriminative ability of coding vectors for better representation and/or classification, and has led to state-of-the-art results in many vision applications. Generally, there are two categories of dictionary learning methods, synthesis and analysis dictionary learning. The paper Blockwise Coordinate Descent Schemes for Efficient and Effective Dictionary Learning by Liu et al. presented a blockwise coordinate descent algorithm for synthesis dictionary learning, while the paper Deep Boosting: Joint Feature Selection and Analysis Dictionary Learning in Hierarchy by Peng et al. extended the discriminative analysis dictionary learning model to a hierarchical deep architecture. Both the two works were evaluated using benchmark image classification datasets. Liu et al. showed that their method can achieve competitive classification performance and much better efficiency compared with other dictionary learning methods. Peng et al. validated the potential of building deep architecture via hierarchical analysis dictionary learning, and their method outperforms state-of-thearts on several visual recognition tasks.

Low level vision is a critical topic in computer vision while smart computing plays an important role. Typical low level vision problems include 3D reconstruction, image enhancement, segmentation, pixel classification, etc. In the paper Multi-view Stereo via Depth Map Fusion: A Coordinate Decent Optimization Method, Li et al. proposed a novel multiview stereo (MVS) method, CoDFusion, by fusing the depth maps from different views. Li et al. adopted the iterative weighted median filtering to solve the $\mathrm{TV}+\mathrm{L} 1$ model, and the experiments validated the effectiveness and efficiency of CoD-Fusion. The paper A Variational based Smart Segmentation Model for Speckled Images by Han et al. studies the segmentation of speckled image. Han et al. suggested a modified variational model together with and alternative direction iteration algorithm to solve it, and tested the efficiency of their method using numerical experiments. The paper Weighted Multifeature Hyperspectral Image Classification via Kernel Joint Sparse Representation by Zhang et al. focuses on pixel classification of hyperspectral images. Zhang et al. used the kernel joint sparse representation method for the fusion of multi-features and the incorporation of contextual neighborhood knowledge. Experiments validated the efficiency and accuracy of their method. 
Emotion and behavior are important factors in human-computer interaction and medical applications, but are challenging to be inferred from visual data. The paper ARCH: Adaptive RecurrentConvolutional Hybrid Networks for Long-Term Action Recognition by Xin et al. studies the long-term action recognition. Xin et al. integrated temporal-spatial-fusion CNN with recurrent network to improve the robustness against spatial and temporal variations, and intra- and inter-class diversities. In the paper Analysis of Physiological for Emotion Recognition with IRS Model, Li et al. investigated the problem of physiological-based emotion recognition, and suggested a Group-based Individual Response Specificity model. A dataset of physiological signals was collected to evaluate the proposed model.

With the fast growth of large scale visual data, hashing has been intensively studied for image and video retrieval. The paper $A$ Fast Binary Encoding Mechanism for Approximate Nearest Neighbor Search by Zhao et al. adopted boosting to generate strong hash function from weak ones. Zhao et al. also showed their method is superior to many existing hashing methods.

We believe that this special would offer a good platform for the readers to find the latest research outputs on the related topics. The guest editors would like to thank all the authors and reviewers for their contributions, and acknowledge the strong support from the Editor-in-Chief of Neurocomputing Journal, Managers Jacqueline Zhu and Sudharshan Raj, and their Elsevier colleagues.

\section{Professor} Lei Zhang

Department of Computing, The Hong Kong Polytechnic University, Hung Hom, Kowloon, Hong Kong E-mail address: cslzhang@comp.polyu.edu.hk
Assistant Professor

Pinar Duygulu

Department of Computer Engineering, Bilkent University, Ankara,

Turkey

E-mail address: duygulu@cs.bilkent.edu.tr

Associate Professor

Wangmeng Zuo

School of Computer Science and Technology, Harbin Institute of Technology, Harbin, China

E-mail address: wmzuo@hit.edu.cn

Professor

Shiguang Shan

Institute of Computing Technology, Chinese Academy of Sciences,

Beijing, China

E-mail address: sgshan@ict.ac.cn

Principal Systems Scientist, Guest Editors

Alex Hauptmann

Principal Systems Scientist, School of Computer Science, Carnegie Mellon University, Pittsburgh, USA E-mail address: alex@cs.cmu.edu

Received 16 October 2015; accepted 16 October 2015

Available online 5 November 2015 